\title{
Heterogeneous Distribution of Neurotensin-like Immunoreactive Neurons and Fibers in the Midbrain Periaqueductal Gray of the Rat
}

\author{
Michael T. Shipley, ${ }^{1,2}$ John H. McLean, ${ }^{1}$ and Michael M. Behbehani ${ }^{3}$ \\ 'Department of Anatomy and Cell Biology, Division of Neurobiology, ${ }^{2}$ Department of Neurosurgery, and ${ }^{3}$ Department of \\ Physiology and Biophysics, University of Cincinnati College of Medicine, Cincinnati, Ohio 45267-0521
}

\begin{abstract}
The midbrain periaqueductal gray (PAG) has been shown to be a site where various manipulations induce pain suppression. Recent physiological evidence (Behbehani and Pert, 1984; Behbehani et al., 1987) suggests that neurotensin has pronounced physiological actions in PAG and effects pain suppression. We have performed immunohistochemical studies in order to determine the magnitude and distribution of neurotensin-like immunoreactive (NT-IR) cell bodies and fibers in PAG. NT-IR cell bodies were common throughout PAG, although there were more in the caudal than the rostral half. NT-IR neurons were much more numerous in the ventral than the dorsal half of PAG, and some appeared to be located within the dorsal raphe nucleus. The pattern of NT-IR fibers was analyzed with the aid of image enhancement/analysis and densitometry. The fibers were found to be heterogeneously distributed, being most heavily concentrated in the region adjacent to the cerebral aqueduct in the caudal twothirds of PAG. The distribution of NT fibers closely matches sites where exogenously applied NT elicits long-lasting excitation of PAG neurons (Behbehani et al., 1987). Based on the known physiological and behavioral actions of NT in PAG, the present anatomical results suggest that NT acts on elements located predominantly in the medial and ventrolateral parts of PAG. Neurons activated by NT may project directly to the nucleus raphe magnus and adjacent ventral medulla (Behbehani and Pert, 1984) to activate neurons that project to the spinal cord and modulate nociceptive circuits.
\end{abstract}

It is well established that stimulation of the midbrain periaqueductal gray (PAG) activates circuits that project to the spinal cord and mediate pain suppression. This phenomenon can be initiated by electrical stimulation (Reynolds, 1969) or local application of the excitatory amino acid glutamate (Behbehani and Fields, 1979). Injection of morphine in PAG also causes long-lasting analgesia, which is blocked by the opiate antagonist naloxone (Yaksh et al., 1976). These manipulations appear to engage circuits in PAG that project to the nucleus raphe magnus (NRM) and adjacent ventral medullary region and activate neurons that project to the dorsal horn of the spinal cord (Basbaum

\footnotetext{
Received May 28, 1986; revised Sept. 23, 1986; accepted Oct. 30, 1986.

We wish to thank Sharon Harding for typing the manuscript and B. Frydel, $M$. Clements, and K. Wilburn for technical assistance. We also wish to thank Dr. Alvin Beitz for his helpful suggestions for optimizing the colchicine concentrations to visualize NT cell bodies. This work was supported by Grants DAMD 17-86C-6005, NS-20643, and NS-23348.

Correspondence should be addressed to Dr. Michael T. Shipley at the above address.
}

Copyright @ 1987 Society for Neuroscience $0270-6474 / 87 / 072025-10 \$ 02.00 / 0$ et al., 1976, 1978; Basbaum and Fields, 1979) and modulate local spinal nociceptive circuits (Basbaum and Fields, 1984). Thus, lesions of NRM and its surrounding area block or attenuate all known forms of PAG-induced analgesia (Behbehani and Fields, 1979; Behbehani and Pert, 1984; Sandkuhler and Gehhart, 1984), and naloxone blocks the analgesia induced by morphine injections in PAG.

Several studies have demonstrated that central administration of the tridecapeptide neurotensin (NT) produces a significant long-lasting analgesia (Clineschmidt and McGuffin, 1977; Nemeroff et al., 1979; Kalivas et al., 1982). Recently, Behbehani and Pert (1984) showed that NT injected in PAG caused pain suppression that was as potent as that induced by similar amounts of morphine. These investigators elucidated several key features of the NT effect: (1) Local application of NT caused long-lasting excitation of PAG neurons; (2) injection of NT in PAG increased the firing rate of NRM neurons having identified projections to the spinal cord; (3) lesions of NRM abolished the antinociceptive action produced by injection of NT in $P \Lambda G$. Thus, it was hypothesized that NT acted upon neurons in PAG that, in turn, activated neurons in NRM ventral medullary area whose projection to the spinal cord is necessary for all known central antinociceptive effects.

Significantly, this NT-activated antinociceptive mechanism, unlike the morphine effect, is not blocked by naloxone. Thus, it would appear that the action of NT is mediated by the same PAG-NRM-spinal cord pathways as morphine but operates through different groups of neurons or different postsynaptic receptor populations. Therefore, it is important to define the neural substrates of NT's action in PAG, as it appears to involve a parallel but independent mechanism to the opiate channel.

The existence of NT in PAG has been established by immunohistochemical methods (Uhl and Snyder, 1976; Kobayashi et al., 1977; Beitz, 1982b; Jennes et al., 1982), and the in vitro binding of tritiated NT in PAG has been demonstrated and interpreted as evidence of the presence of NT receptor sites (Lazarus et al., 1977; Young and Kuhar, 1981; Quirion et al., 1982). However, these previous reports provide little information about the topological organization of neurotensinergic elements in PAG. The present study was undertaken to close this gap by characterizing the distribution of NT-immunoreactive (IR) neurons and fibers in PAG. The results indicate that NT fibers have a markedly heterogeneous distribution in PAG.

\section{Materials and Methods}

Adult, male Sprague-Dawley rats (Harlan and Zivic-Miller) were used. The animals were deeply anesthetized with sodium pentobarbital and transcardially perfused. Following a brief clearing rinse with normal 
saline, the animals were perfused for $30 \mathrm{~min}$ with $4 \%$ paraformaldehyde in sodium phosphate buffer $(\mathrm{PB})$. The brains were removed, postfixed for $30 \mathrm{~min}$ to $5 \mathrm{hr}$, and then placed in $20 \%$ sucrose-PB on a shaker table in a cold room $\left(4^{\circ} \mathrm{C}\right)$ overnight. Frozen sections $(30 \mu \mathrm{m}$ thick) were collected in cold PBS, rinsed $(2 \times 10 \mathrm{~min})$, and transferred to PBS containing $2 \%$ normal goat serum (NGS) and $0.3 \%$ Triton $X-100$ for $45 \mathrm{~min}$. Following a PBS rinse $(2 \times 10 \mathrm{~min})$, the sections were incubated overnight at $4^{\circ} \mathrm{C}$ in rabbit anti-NT antisera (Immuno Nuclear, Inc.; diluted 1:500) in PBS containing 2\% NGS and 0.3\% Triton. The sections were then rinsed $(2 \times 10 \mathrm{~min}$ PBS) and incubated for $45 \mathrm{~min}$ in biotinylated goat anti-rabbit IgG (Vector Lab) in PBS containing $2 \%$ NGS and $0.3 \%$ Triton. Following 2 rinses $(2 \times 10 \mathrm{~min}$ PBS $)$, the sections were incubated in avidin-biotin complex (ABC) (Vector Lab) in PBS containing $2 \%$ NGS. Usually, the biotinylated IgG-rinse-ABC cycle was repeated a second time. After the $A B C$ step the sections wcre rinscd $(2 \times 10 \mathrm{~min})$ in phosphate buffer and then incubated in a solution containing $0.05 \%$ diaminobenzidine and $0.01 \% \mathrm{H}_{2} \mathrm{O}_{2}$ in phosphate buffer for 5-20 min. The DAB reaction was stopped in 3 rinses $(10 \mathrm{~min}$ each) of phosphate buffer and mounted onto gelatinized slides.

In most cases, the staining was intensified by first defatting the mounted sections in a graded series of ethyl alcohol, followed by rehydration and a brief $(1-2 \mathrm{~min})$ reaction with $1 \%$ osmium tetroxide. The sections were then cleared and coverslipped with Permount.

Immunohistochemical controls were performed by incubating some sections with the primary antibody omitted or with the primary antibody preabsorbed by an excess of NT (Immuno Nuclear, Inc.; $50 \mu \mathrm{g}$ / $\mathrm{ml}$ of diluted antisera). In no case was immunocytochemical staining observed in the PAG control sections.

Cell body versus fiber staining. During the course of this study, it became clear that different procedures were required for optimal staining of fibers and cell bodies. For cell bodies, pretreatment with colchicine was obligatory and the dose was critical. Optimal cell body staining was achieved with $50 \mu \mathrm{g} / 10 \mu \mathrm{l}$ colchicine delivered intracerebroventricularly $48 \mathrm{hr}$ prior to sacrifice ( $n=4$ rats); increasing colchicine to $200-400$ $\mu \mathrm{g} / 10 \mu \mathrm{l}$ decreased the number of positively stained neurons $(n=2$ rats). Omission of colchicine pretreatment altogether led to optimal fiber staining ( $n=8$ rats). In all other respects, the immunohistochemical procedures were identical for fiber and cell body material.

Image enhancement/analysis. The distribution of NT-IR fibers was analyzed with the aid of video-computer-based microscopy. The sections were viewed by dark- and bright-field microscopy, and the images were captured by a Newvicon video camera. The camera output was Icd through a real-time video processor (Image $\Sigma$-Model 794, Hughes Aircraft Company), which provided an initial stage of contrast enhancement, to the input of an image-analysis computer (Magiscan 2A-Joyce Loebl/Nikon).

Two methods were developed to analyze the organization of NT-IR fibers in PAG.

1. Color-coded density maps of NT staining were generated at several rostrocaudal levels through PAG. In these maps, the areal density of NT-IR fiber staining was represented by different colors. An image of PAG containing NT-IR fibers was digitized into 64 discrete gray levels. Using a threshold function, the range of levels encompassing the highest to the lowest densities of immunoreactive fibers was determined. The densities in this range were assigned colors from a scale in which blue represented the lowest density followed by green, yellow, orange, and red for increasing density. White was assigned to the highest density range to highlight regions containing the highest concentrations of NTIR fibers. Identical conditions of illumination, gray-level thresholding, and color assignment were applied to a series of rostral to caudal coronal sections through PAG taken from one representative case in which the section-to-section variation in background staining was negligible. Care was taken to use only those specimens for which a preliminary measurement and microdensitometric sampling indicated that variations in section thickness were negligible.

It is important to note that these density maps are intended only to compare the relative distribution of NT-IR fibers at different rostrocaudal levels of PAG. From such data it is not possible to estimate the absolute amounts of NT present because the densities reflect not only variations in NT-IR, but also a variety of specific immunocytochemical parameters ranging from the degree of fixation to the duration of the DAB chromogenic reaction.

2. An isodensitometry program was developed and used to provide quantitative measures of the distribution of staining densities in objectively delineated subregions of PAG.
Thus, as will be described below, different parts of PAG characteristically have different densities of NT-IR staining. The function of the isodensitometry program is to calculate the gray levels (0-63) (staining intensities) of each of the $512 \times 512$ points in the digitized image and convert the distribution of gray values to a density scale $(0.0-1.0)$. The program then draws closed boundary lines around contiguous regions of equal density (isodensity contours). Since the range of densities within any local region may fluctuate somewhat because of the discrete nature of the stain, it is useful to lump densities within certain ranges together, and the program allows the user to specify the number and cut-off points of these isodensity regions. The program then delineates, using different colored binary overlay lines, the boundaries of the regions whose densities fall within specified ranges. A recursive procedure identifies lowdensity regions surrounded by higher-density regions so that "holes in the donut" are detected. The result of these operations is to delineate regions of isodensity in the image. The user may then use different conventions (solid filling, dots of different spacing, etc.) to indicate different regions of isodensity so that the information can be reduced to a black-and-white schematic. The numerical value of the average density for each isodensity region can also be represented in the graphic printout. By using the same density cut-off values and illumination conditions and by controlling for unacceptable variations in section thickness, successive sections can be analyzed to generate isodensity maps for a series of sections.

The same qualifications stated for the color density maps apply to the isodensitometric analysis. Thus, the density readings have no simple relationship to the amount of NT present in the measured regions, although the ratios of densities in different regions do reflect the relative distribution of the peptide within the limits of sensitivity of immunohistochemistry. The value of such an analysis is that it is possible to estimate that one subregion of PAG has, for example, twice the fiber density as another.

\section{Results}

Appearance of NT-IR fibers

In cases without colchicine treatment, NT immunoreactivity in PAG was localized exclusively in varicose fibers and isolated varicose profiles (Fig. 1, $B, C$ ). These isolated varicosities were of the same size and shape as the fiber-linked varicosities and are here interpreted as NT-IR terminals. As will be discussed presently, the density of these fibers and varicosities varies systematically in different parts of PAG. In certain regions, e.g., mid-rostrocaudal circumaqueductal parts of PAG, the density of stained profiles was very high. Paradoxically, despite their density, these fibers and varicosities were difficult to stain; best results were obtained with the use of the "double" ABC method, poststaining intensification with osmium tetroxide, and considcrable trial and crror to optimize the fixative-staining protocol. In several cases, however, excellent staining was achieved with the fibers and varicosities stained dark black against a pale beige background (Fig. 1).

\section{Organization of NT-like fibers in $P A G$}

NT-IR fibers and varicosities were heterogeneously distributed in PAG (Fig. 1 $A$ ). The density of the fibers varied both along the rostrocaudal axis and within the coronal plane at all rostrocaudal levels. NT-IR fibers were more numerous in the caudal two-thirds of PAG, being most abundant at mid-rostrocaudal levels.

Figure 2 shows a series of rostral-to-caudal color-coded fiber density maps through PAG. The first level depicted (Fig. $2 A$ ) is at the rostral end of the cerebral aqueduct. NT-IR activity is confined mainly to a narrow juxtaqueductal zone and is of low to moderate density. The next section (Fig. $2 B$ ) is at about the level of the rostral-most cells of the mesencephalic nucleus of V. NT-IR fibers are more abundant and are densest along the lateral walls of the aqueduct. The fibers extend more exten- 

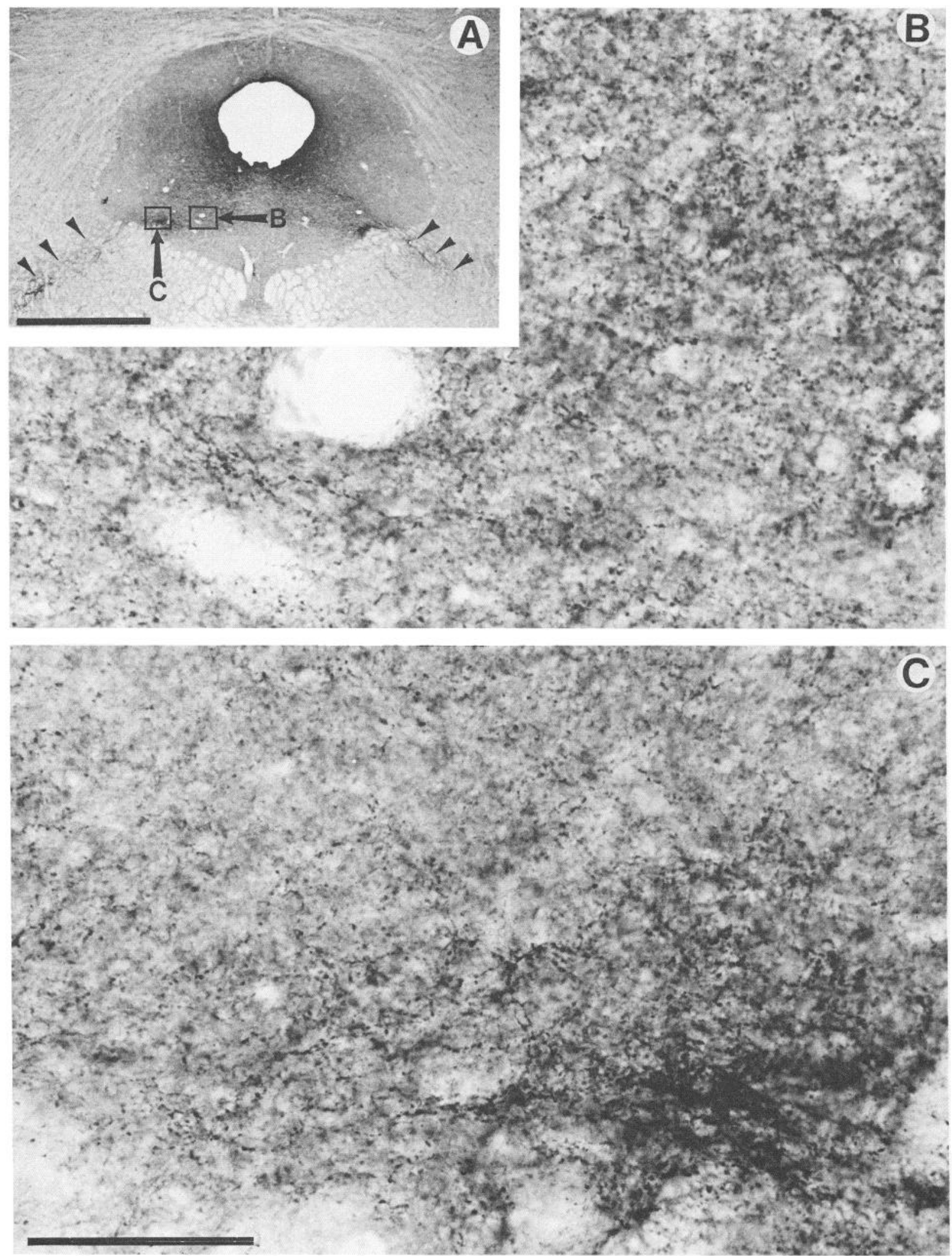

Figure 1. Bright-field photomicrographs showing the distribution of NT-IR fibers in PAG. A, Low-magnification photomicrograph provides an overview of NT-IR fibers that stain darkly against a pale background. Fiber bundles (arrowheads) extend from the ventrolateral corner of PAG. $B$ and $C$, High-magnification photomicrographs from moderately $(B)$ and densely stained $(C)$ regions shown in $A$. Most NT immunoreactivity is seen as varicosities, although varicose axonal profiles are also visible. Scale bar: $A, 1 \mathrm{~mm} ; C, 50 \mu \mathrm{m}(B$, same magnification as $C)$. 

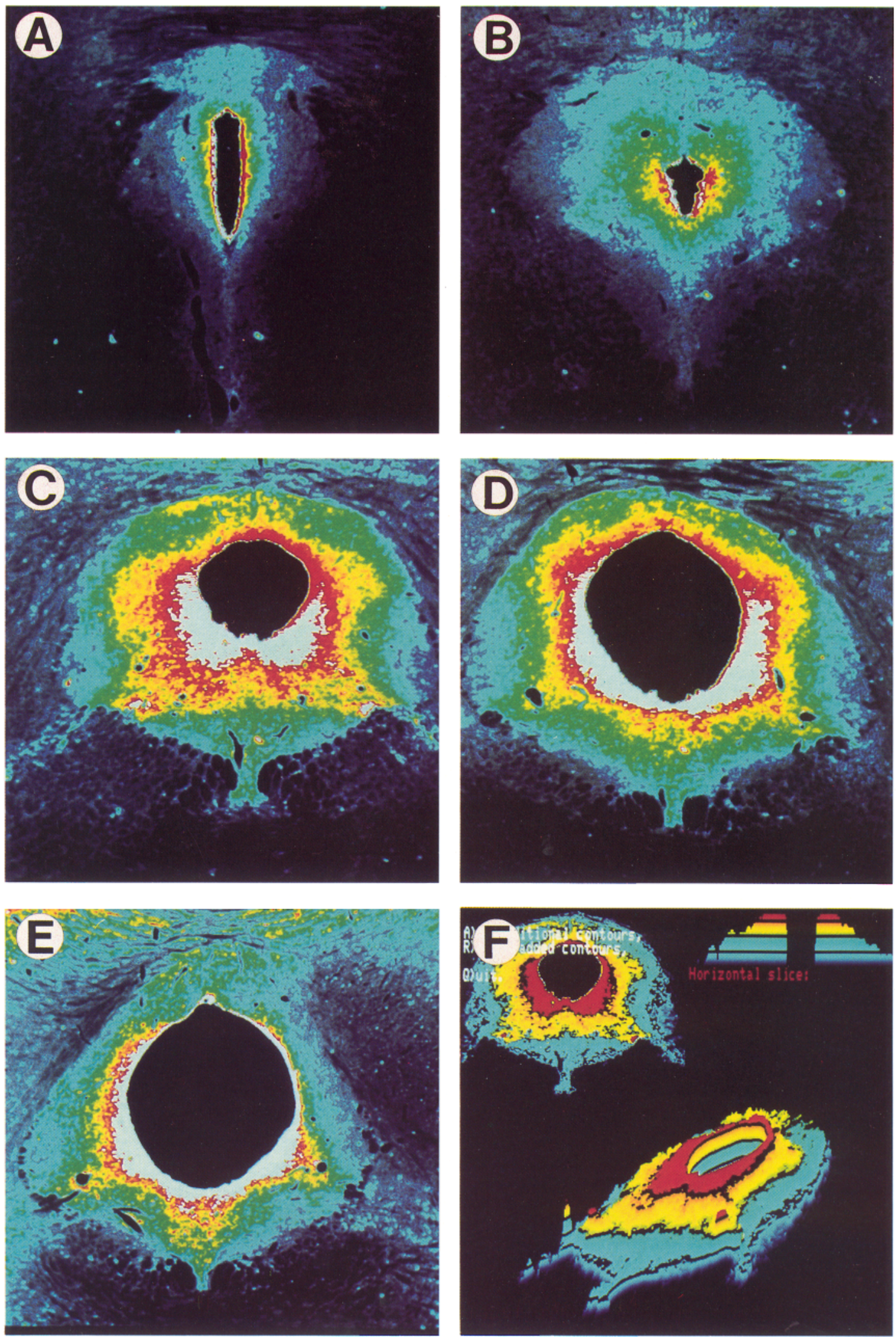

Figure 2. Color-coded, image-enhanced micrographs of sections through rostral $(A)$ to caudal $(E)$ levels of PAG. The colors represent density gradients of NT-IR fibers, as explained in the text. $F$, Color-coded image from the section shown in $C$ and Figure 1, with 3-dimensional perspective added. This aids the visualization of the heterogeneity of NT staining. 


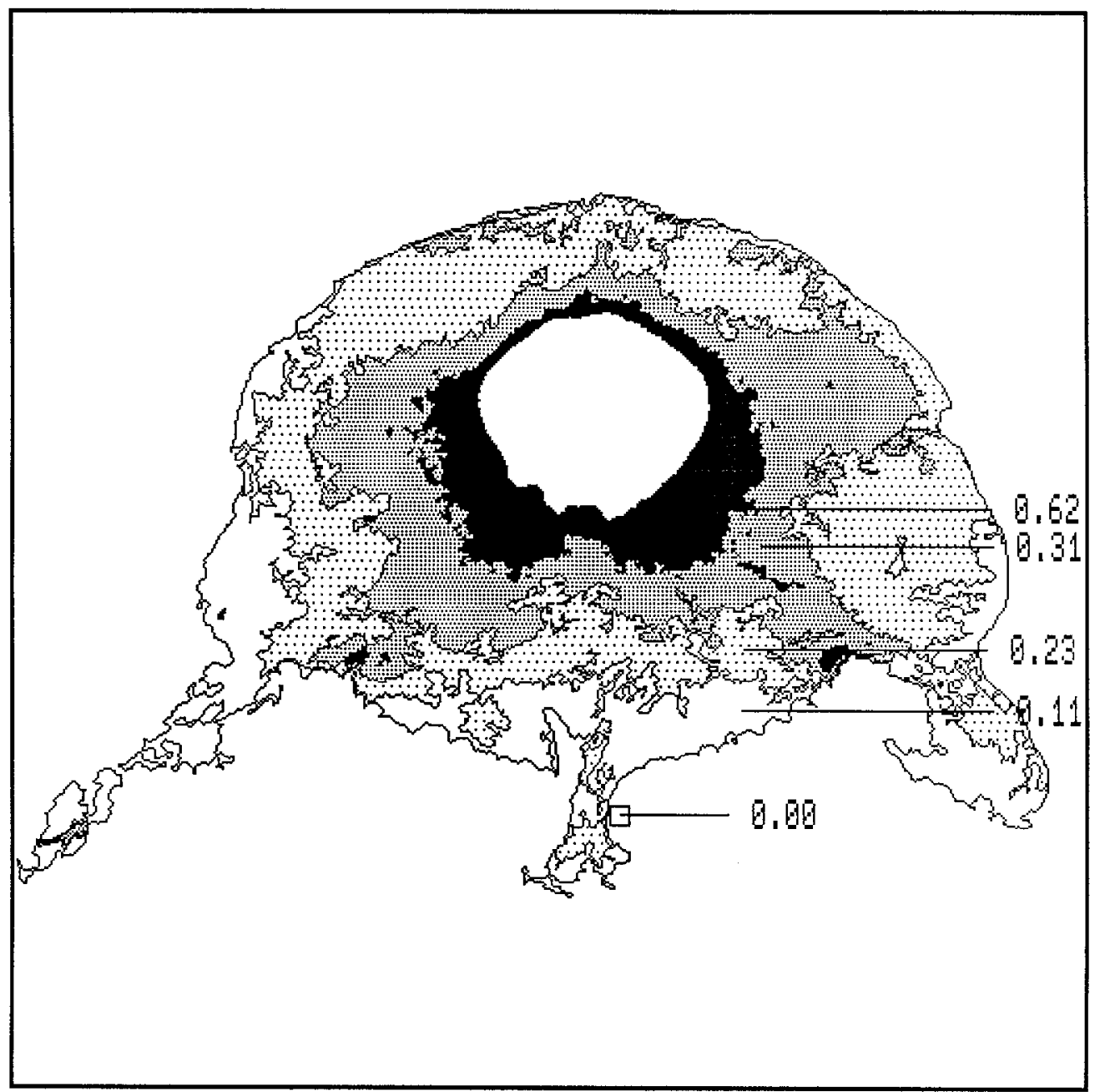

Figure 3. Computer-aided drawing displaying the relative distribution and density of NT fibers in PAG. The juxtaqueductal region has densest representation, with approximately 6 times immunoreactivity than ventral regions of PAG at this level. The section analyzed is the same as that displayed in Figures 1 and $2, C$ and $F$. sively into the ventral midline isthmus of PAG than in the previously described rostral section.

At the next caudal level (Fig. 2C) NT-IR fibers achieve their maximal extent and density. At least some fibers are present throughout all parts of PAG. The maximum density is still in the lateral and ventral juxtaqueductal regions, but the density throughout the central two-thirds core of PAG is nearly as great as it was in the juxtaqueductal zones in the more rostral sections. Fibers extend into the ventral isthmus-dorsal raphe region, where they reach moderately high densities. Also conspicuous at this level is intense bilateral NT-IR staining in a ventrolateral to dorsomedially oriented strip that joins PAG at its ventrolateral wings. These strips correspond to bundles of NT-IR fibers (Fig. $1 A$ ) and were observed at this same rostrocaudal level in every case. They appear to comprise fascicles of NT-IR fibers entering and/or exiting PAG.

In the next caudal section (Fig. $2 D$ ), the aqueduct has enlarged almost 2-fold, and the level is at the beginning of the caudal third of PAG. The extent and density of NT-IR fibers is only slightly less than, and has a similar pattern to, the previously described rostral section. The dorsolateral juxtaqueductal parts of PAG are somewhat more heavily innervated than at more rostral levels. In general, however, the density of NT-IR fibers is still greatest in the cental juxtaqueductal core of PAG, with the highest density around the ventrolateral walls of the lumen.

At the next caudal level (Fig. 2E), NT-IR fibers are still concentrated in the central core of PAG, and there is an increased density in the ventromedial part of PAG. Overall, the density of fibers is lower than at mid-rostrocaudal levels of PAG.

To provide a better appreciation of the gradient of NT-IR densities, Figure $2 F$ shows the same section as illustrated in Figure $2 C$ with the densities represented as amplitude modulations in a pseudo-3D contour plot.

\section{Microdensitometry}

From the foregoing account it is clear that NT-IR fibers are most heavily concentrated in the middle and caudal parts of PAG. Moreover, at all rostrocaudal levels the fibers are preferentially distributed in the central juxtaqueductal core of PAG. Microdensitometric measures confirmed the existence of this heterogeneity and provided information about the relative density of fibers in different parts of the coronal plane.

Figure 3 shows the results of a microdensitometric analysis of the most heavily innervated coronal level, which is the same section depicted in Figures 1 and 2, $C$ and $F$. The measurement adjacent to the ventral isthmus of PAG was taken as the zero background reading because no NT-IR staining was found in this zone. Isodensity contour lines in PAG were plotted as de- 


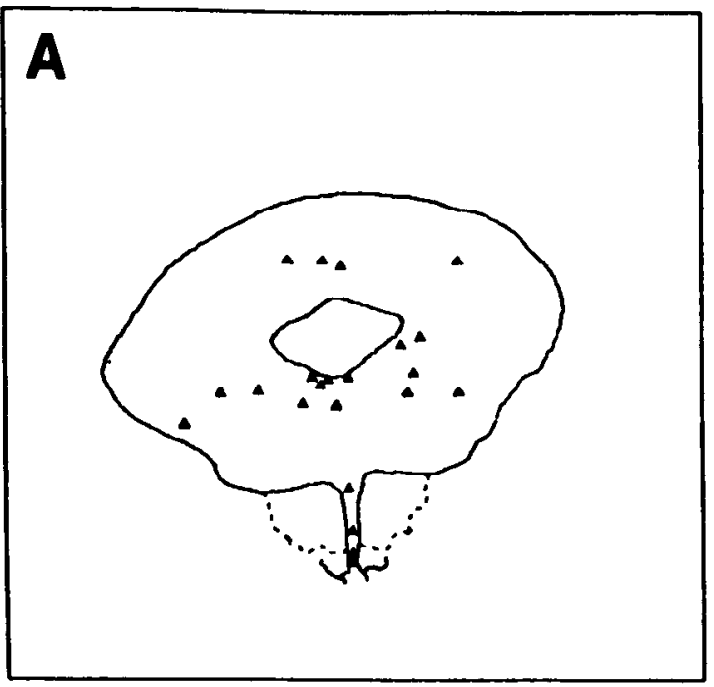

B

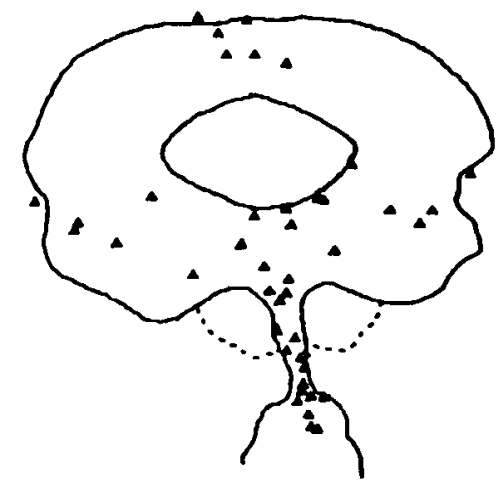

\section{C}

D
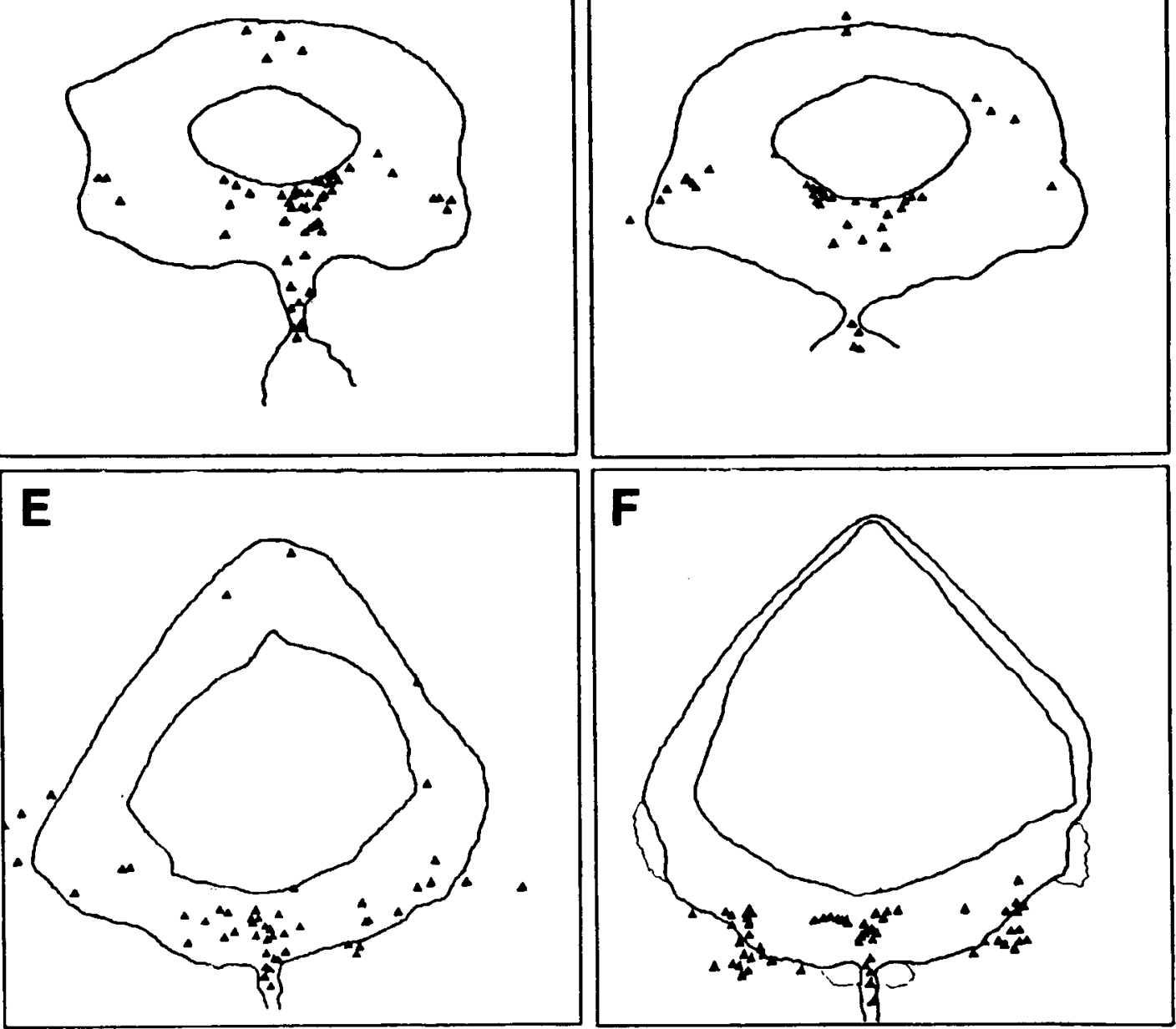

Figure 4. Computer-assisted drawings displaying the distribution of NT-IR neurons taken from rostral $(A)$ to caudal $(F)$ levels of PAG in one rat.

scribed in Materials and Methods. The region of highest density near the ventrolateral aqueduct has an average density of 0.62 ; by contrast, the peripheral parts of PAG and the region of the ventral midline isthmus have a density of 0.11 , implying that the juxtaqueductal zone contains roughly 6 times the density of fibers per unit area as does the isthmus, which is consistent with the impression obtained by direct high-magnification observation. 

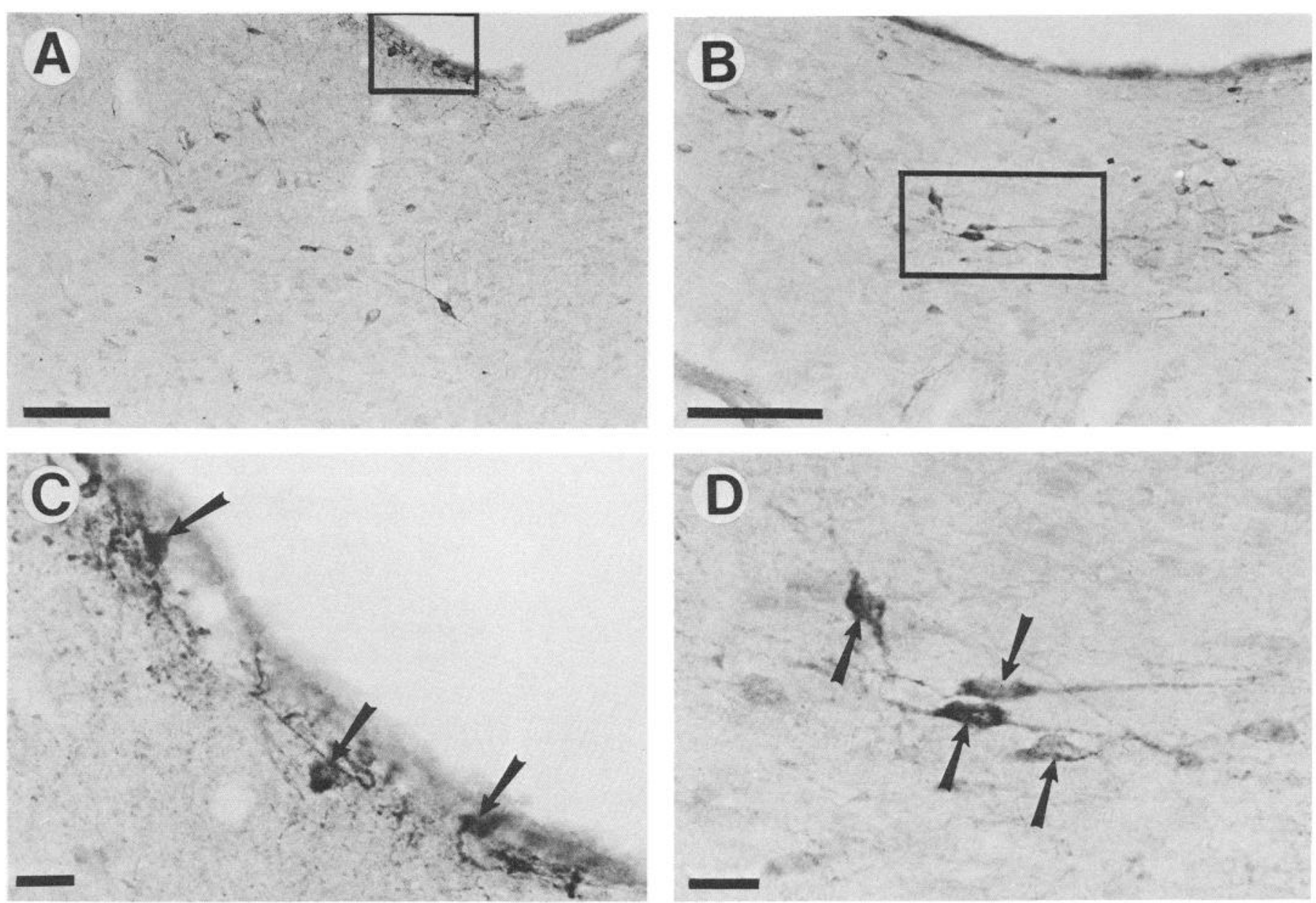

Figure 5. Bright-field photomicrographs of middle $(A, C)$ and caudal $(B, D)$ levels of PAG showing portions containing NT-IR cell bodies. In $A$ and $B$ numerous immunoreactive cells (arrows) are observed among many more unreactive neurons. Panel $C$ is higher magnification view of region displayed in $A$. Note the small immunoreactive cells (arrows) located next to the cerebral aqueduct ( $A q$ ). Panel $D$ is a higher magnification of the bounded area in $B$. The immunoreactive neurons here appear to be spherical and perhaps bipolar. Scale bar: $A$ and $B, 200 \mu \mathrm{m} ; C$ and $D, 20 \mu \mathrm{m}$.

\section{$N T$-IR cell bodies in $P A G$}

As with NT-IR fibers, NT-IR cell bodies were heterogeneously distributed in PAG (Fig. 4). In general, more NT-IR neurons were present in the caudal than the rostral half of PAG. At all rostrocaudal levels, there were many more NT-positive neurons in the ventral than the dorsal half of the central gray. Although less pronounced, there was a further tendency for neurons to be more numerous near the aqueduct. Neurons at the extreme lateral or ventrolateral edge of PAG, however, were not uncommon. At most rostrocaudal levels (Fig. 4, $A-F$ ) a significant proportion of the positive neurons were located in the ventral midline portion of PAG corresponding to the ventromedian subdivision (Steinbusch and Nieuwenhuys, 1983) of the dorsal raphe nucleus. At the most caudal levels of PAG (Fig. 4, E, F), the neurons were mainly clustered in 2 groups: the aforementioned ventromedian subdivision of the dorsal raphe and the ventromidlateral portion of PAG. NT-IR neurons averaged 13.5 $\mu \mathrm{m}$ in long axis (range, 8-20 $\mu \mathrm{m}$ ) (Fig. 5). Juxtaqueductal neurons were common in the ventral portion around the aqueduct and were slightly smaller than other PAG NT-IR neurons, averaging $11 \mu \mathrm{m}$ in long axis. Juxtaqueductal neurons were often bipolar in appearance, and their dendrites were orientated parallel to the surface of the cerebral aqueduct. Neurons located in ventromedial, lateral, and dorsal positions of PAG were sometimes bipolar in appearance but were usually spherical with 34 dendrites extending from the soma.

\section{Discussion}

The major result of this study is that NT-IR neurons and especially fibers have a dense but heterogeneous distribution in the midbrain periaqueductal gray. Coupled with previous reports that NT injected into PAG causes a potent, long-lasting, naloxone-insensitive, raphe magnus-dependent pain suppression (Clineschmidt and McGuffin, 1977; Nemeroff et al., 1979; Behbehani and Pert, 1984), the present results suggest that NT acts upon discrete, localized PAG circuits to activate a PAGNRM-spinal cord antinociceptive mechanism.

Analysis of the immunocytochemical data reported here indicates that NT-IR fibers are most heavily concentrated in the central core of PAG, particularly in the region adjacent to the ventrolateral and ventral walls of the cerebral aqueduct. More fibers are present in the middle and caudal parts of PAG than in the rostral third of the structure.

Even within the regions of PAG that contain NT-IR fibers, there is considerable heterogeneity in the abundance of the fibers. The highest fiber density is in the ventrolateral juxt- 
aqueductal region, which may contain at least 6 times the number of fibers as more peripheral parts of PAG. The fact that NTIR fibers are densest around the cerebral aqueduct may explain why this peptide has a potent antinociceptive action after intracerebroventricular application.

There is an interesting relationship between the distribution of NT-IR fibers and the cytoarchitecture of PAG. Opinion is divided on whether the cellular architecture of PAG comprises subdivisions. On the one hand, Mantyh (1982: rat, cat, monkey) and Gioia et al. (1984: cat) concluded that the variations in the cytology of PAG are not significant enough to warrant the postulation of subdivisions of this structure. On the other hand, Hamilton (1973) and, more recently, Beitz and Shepard (1985) concluded that there are systematic cytoarchitectonic variations in PAG, the latter authors using morphometric and cluster analysis to support their parcellations. We have not attempted a comprehensive reanalysis of the Nissl architecture of P $\Lambda G$ of the rat in the present study, but it is our impression that the subdivisional schemes advanced by Hamilton and Beitz are supportable. The heterogeneous distribution of NT-IR fibers reported here is also consistent with the existence of subdivisions in PAG. The densest fiber staining is localized in the circumaqueductal part of PAG, which corresponds to the medial division of PAG described by both Hamilton (cat: 1973) and Beitz (rat: 1985). The overall distribution of NT-IR fibers, in fact, corresponds remarkably well to the outlines of the feline medial division as defined by Hamilton. The medial subdivision of Beitz and Shepard (1985) in the rat lacks the ventrolateral extensions of Hamilton's (1973) medial subdivision in the cat, but the distribution of NT-IR fibers in the rat shows similar ventrolateral winglike extensions. This minor difference notwithstanding, the present observations on the distribution of a peptide with demonstrated physiological actions in PAG would seem to correspond well to and hence support the existence of subdivisions in PAG. Moreover, since the preferentially medial and ventral localization of NT-like fibers corresponds well to the locations of neurons that are potently excited by NT in an in vitro preparation (Behbehani et al., 1987), the present finding suggests that the proposed cytological subdivisions of Hamilton (1973) and Beitz (1985) may have functional significance.

The only previous description of the distribution of NT-IR fibers in PAG was in the survey study by Jennes et al. (1982), and the localization data reported here are in agreement with their comments on NT in PAG. The localization of numerous other transmitter markers in PAG has been described, and these findings have been discussed in recent papers (Basbaum and Fields, 1984; Beitz, 1985), so here we will only briefly summarize the distribution of other markers in relationship to NT. Of the transmitter markers studied to date, it would appear that fibers and terminals are distributed in 3 general patterns: fiber systems that are preferentially distributed in the (1) medial or (2) peripheral parts of PAG, or (3) fiber systems that are distributed more or less uniformly throughout the central gray.

The majority of the neurochemically defined fiber systems are distributed preferentially in the medial subdivision of PAG and include cholecystokinin-8 (Kiyama et al., 1983), FMRFamide (Triepel and Grimmelikhuijzen, 1984), ACTH (Romagnano and Joseph, 1983), substance P (Moss and Basbaum, 1983), and leu-enkephalin (Moss et al., 1983). Somatostatin fibers, however, are preferentially distributed in the peripheral parts of PAG (Finley et al., 1981); serotoninergic (Clements et al., 1985) and histaminergic fibers (Watanabe et al., 1984) ap- pear to be uniformly distributed in PAG. The presence of other transmitter markers in PAG has been reported, including dynorphin (Khachaturian et al., 1982) and enkephalin (met-, leuforms not distinguished) (Uhl et al., 1979) but without definitive information on the pattern of distribution within PAG. The present findings and the earlier survey study by Jennes et al. (1982) are in agreement that NT-IR fibers are primarily localized to the medial subdivision of PAG, especially as parcellated by Hamilton (1973) in the cat. Thus, it would appear that the majority of transmitter-specific fiber systems studied to dateincluding all of the neuropeptides, except for somatostatin-are preferentially localized in the medial subdivision and/or its ventrolateral wings. Thus, the medial and ventrolateral parts of PAG may comprise a zone that integrates information from multiple peptidergic systems. By contrast, retrograde labeling studies (Beitz, 1982b; Beitz et al., 1983a, b) indicate that neurons projecting out of $P \wedge G$ tend to be located in more peripheral portions of PAG. Taken together, these results suggest the possibility that central portions of PAG may be more involved in integrative activity, whereas more peripheral portions of the structure may be more involved in output functions. Such a scheme may be overly simplistic in view of the fact that some peripherally located neurons extend dendrites into the more medial zones of PAG (Beitz and Shepard, 1985). In addition, there is surprisingly little information on the precise termination patterns of afferents to PAG, so it is not clear whether extrinsic afferents to PAG terminate preferentially in the central, peptiderich area and there are few systematic studies of the distribution of afferent inputs and efferent neurons along the rostrocaudal axis of PAG. Nonetheless, the possibility that the PAG is comprised of predominantly afferent-integrative and efferent zones may be useful to bear in mind as future anatomical studies disclose more of the organization of this important structure.

The existence of NT-IR neurons in PAG was initially reported by Jennes et al. (1982). This finding was confirmed and the distribution of NT-IR neurons was described in greater detail by Beitz and coworkers (Beitz et al., 1983b). To this, the present study adds information about the distribution of NT-IR neurons along the rostrocaudal axis of PAG, indicating that at more caudal levels the neurons tend to become progressively localized in the ventral parts of PAG. In addition, we draw attention to the existence of numerous NT-IR neurons immediately adjacent to the cerebral aqueduct. These juxtaqueductal neurons were not noted in previous reports. They appear to differ in shape and possibly in size from other PAG NT neurons and may, thus, constitute a functionally distinctive subpopulation. In general, however, our observations are in good agreement with earlier descriptions (Jennes et al., 1982; Beitz et al., 1983b).

NT-IR neurons are generally most numerous at the same rostrocaudal levels that contain the highest density of NT-like fibers, and there is a further, though less pronounced, tendency for such neurons to be located in or near the PAG subregions richest in NT-IR fibers. It would seem reasonable, therefore, to suggest that local neurotensinergic neurons contribute significantly to the fiber and terminal staining in PAG. The fiber staining picture may contain some contribution from the dendrites of these NT neurons, but several observations suggest that this contribution is probably small. In none of the colchicinetreated animals were the dendrites of NT neurons stained far beyond their initial segments, and, when visible, the dendrites appeared to be fairly thick, distinctive processes. Examination of the fiber staining in non-colchicine-treated cases did not re- 
veal such thick dendrite-like processes. In point of fact, one of the initial obstacles in this study was that the NT-IR fibers and varicosities were of such a fine caliber that osmium intensification was required to visualize them. Under these conditions, the fibers had the appearance of preterminal axons: small diameter and numerous varicosities. Thus, while it is possible that the dendrites of local NT neurons contribute somewhat to the fiber density maps, we suggest that this contribution is probably minimal and that most of the fiber staining is associated with terminal and preterminal axons.

On the basis of the present observations, therefore, we hypothesize that the chemoanatomical substrate for NT's antinociceptive action in PAG is due, at least in part, to the release of the peptide from terminals of local PAG neurotensinergic neurons onto other PAG neurons. The identity of the neurotensinoceptive neurons remains to be determined, as well as whether they project directly to the NRM or via other PAG neurons. Whether direct or indirect, the linkage between PAG neurons reactive to NT and neurons in NRM must be fairly secure because Behbehani and Pert (1984) showed that NRM neurons with identified projections to the spinal cord are activated when NT is injected into PAG.

An important question is whether, in addition to these hypothesized NT local neuronal circuits, there are also extrinsic NT afferents to PAG. The present experiments do not address this issue directly except that we did observe fascicles of NTlike fibers along the ventrolateral borders of PAG. These fascicles may be axons of PAG-NT neurons that exit PAG since Beitz (1982b) has demonstrated that some NT-IR neurons in PAG send axons to NRM. Additionally, these fascicles may contain axons of NT-IR fibers from extrinsic afferent sources. There are reports of NT-IR neurons in several forebrain and brain-stem areas (Jennes et al., 1982) known to project to PAG (Beitz, 1982a). The use of combined immunohistochemical and retrograde tracing techniques may be able to resolve whether there are extrinsic NT afferents. The existence of extrinsic NT inputs would make it necessary to consider parallel (local and extrinsic) neurotensinergic channels in PAG; the lack of extrinsic NT afferents would suggest that the antinociceptive actions of this peptide are regulated exclusively by a convergence of afferent inputs upon local NT neurons in PAG. It is also conceivable that NT neurons influence both PAG and NRM, as Beitz has shown that some neurons in PAG labeled by retrograde transport of HRP placed in NRM are also immunoreactive for NT (Beitz, 1982b).

The results of the present study, and the in vitro experiments reported in the companion article, provide the basis of suggesting a minimal neural substrate for NT-PAG pain suppression: PAG contains NT-IR neurons whose axons probably arborize appreciably in PAG. These NT terminals are heterogeneously distributed, being densest in the medial subdivision of the caudal two-thirds of PAG. Neurons in this same subregion of PAG are strongly excited by exogenously applied NT (Behbehani et al., 1987); this excitatory effect is unaffected by calcium removal, indicating that the peptide is acting at postsynaptic sites (Behbehani et al., 1987). It is reasonable, therefore, to suggest that the PAG neurons postsynaptic to NTcontaining terminals project to the NRM and adjacent medulla, where they cause the activation of neurons that mediate pain suppression at the spinal cord level. The NT antinociceptive mechanism may thus operate via the NT-PAG circuits suggested here and by parallel circuits involving direct projections from
PAG-NT neurons to NRM (Beitz, 1982b). The elucidation of the physiological conditions that activate neurotensinergic-PAGNRM circuits and identification of the specific PAG and NRM neurons that receive NT synapses are important issues for further study.

\section{References}

Basbaum, A. I., and H. L. Fields (1979) The origin of descending pathways in the dorsolateral funiculus of the spinal cord of the cat and rat: Further studies on the anatomy of pain modulation. J. Comp. Neurol. 187: 513-532.

Basbaum, A. I., and H. L. Fields (1984) Endogenous pain control systems: Brainstem spinal pathways and endorphin circuitry. Annu. Rev. Neurosci. 7: 309-338.

Basbaum, A. I., C. H. Clanton, and H. L. Fields (1976) Opiate and stimulus-produced analgesia: Functional anatomy of medullospinal pathway. Proc. Natl. Acad. Sci. USA 73: 4685-4688.

Basbaum, A. I., C. H. Clanton, and H. L. Fields (1978) Three bulbospinal pathways from the rostral medulla of the cat. An autoradiographic study of pain modulating systems. J. Comp. Neurol. 178: 209-224.

Behbehani, M. M., and H. L. Fields (1979) Evidence that an excitatory connection between the periaqueductal gray and nucleus raphe magnus mediates stimulation produced analgesia. Brain Res. 170: 85-93.

Behbehani, M. M., and A. Pert (1984) A mechanism for the analgesic effect of neurotensin as revealed by behavioral and electrophysiological techniques. Brain Res. 324: 35-42.

Behbehani, M. M., M. T. Shipley, and J. H. McLean (1987) Effect of neurotensin on neurons in the periaqueductal gray: An in vitro study. J. Neurosci. 7: 2035-2040.

Beitz, A. J. (1982a) The organization of afferent projections to the midbrain periaqueductal gray of the rat. Neuroscience $7: 133-159$.

Beit, A. J. (1982b) The sites of origin of brain stem neurotensinergic and serotonergic projections to the rodent nucleus raphe magnus. $J$. Neurosci. 2: 829-842.

Beitz, A. J. (1985) The midbrain periaqueductal gray in the rat. I. Nuclear volume, cell number, density, orientation, and regional subdivisions. J. Comp. Neurol. 237: 445-449.

Beitz, A. J., and R. D. Shepard (1985) The midbrain periaqueductal gray in the rat. II. A Golgi analysis. J. Comp. Neurol. 237: 460-475.

Beitz, A. J., M. A. Mullett, and L. L. Weiner (1983a) The periaqueductal gray projections to the rat spinal trigeminal, raphe magnus, gigantocellular pars alpha and paragigantocellular nuclei arise from separate neurons. Brain Res. 288: 307-314.

Beitz, A. J., R. D. Shepard, and W. E. Wells (1983b) The periaqueductal gray-raphe magnus projection contains somatostatin, neurotensin, and serotonin but not cholecystokinin. Brain Res. 261: 132137.

Clements, J. R., A. J. Beitz, T. F. Fletcher, and M. A. Mullett (1985) Immunocytochemical localization of serotonin in the rat periaqueductal gray: A quantitative light and electron microscopic study. J. Comp. Neurol. 236: 60-70.

Clineschmidt, B. V., and J. C. McGuffin (1977) Neurotensin administered intracisternally inhibits responsiveness of mice to noxious stimuli. Eur. J. Pharmacol. 46: 395-396.

Finley, J. C. W., J. L. Maderdrut, L. J. Roger, and P. Petrusz (1981) The immunocytochemical localization of somatostatin-containing neurons in the rat central nervous system. Neuroscience 6: 21732192.

Gioia, M., R. Bianchi, and G. Tredici (1984) Cytoarchitecture of the periaqueductal gray matter in the cat: A quantitative Nissl study. Acta Anat. 119: 113-117.

Hamilton, B. L. (1973) Cytoarchitectural subdivisions of the periaqueductal gray matter in the cat. J. Comp. Neurol. 149: 1-28.

Jennes, L., W. E. Stumpf, and P. W. Kalivas (1982) Neurotensin: Topographical distribution in rat brain by immunohistochemistry. $\mathrm{J}$. Comp. Neurol. 210: 211-224.

Kalivas, P. W., B. Gau, C. B. Nemeroff, and A. J. Prange, Jr. (1982) Antinociception after microinjection of neurotensin into the central amygdaloid nucleus of the rat. Brain Res. 243: 279-286.

Khachaturian, H., S. J. Watson, M. E. Lewis, D. Coy, A. Goldstein, and H. Akil (1982) Dynorphin immunocytochemistry in the rat central nervous system. Peptides 3: 941-954.

Kiyama, H., S. Shiosaka, Y. Kubota, H. J. Cho, H. Takagi, K. Tateishi, 
E. Hashimura, T. Hamaoka, and M. Tohyama (1983) Ontogeny of cholecystokinin- 8 containing neuron system of the rat: An immunohistochemical analysis-II. Lower brain stem. Neuroscience 10: 1341-1359.

Kobayashi, R. M., M. Brown, and W. Vale (1977) Regional distribution of neurotensin and somatostatin in rat brain. Brain Res. 126: $584-588$

Lazarus, L. H., M. R. Brown, and M. H. Perrin (1977) Distribution, localization and characteristics of neurotensin binding sites in the rat brain. Neuropharmacology 16: 625-629.

Mantyh, P. W. (1982) The midbrain periaqueductal gray in the rat, cat, and monkey: A Nissl, Weil and Golgi analysis. J. Comp. Neurol. 204: 349-363.

Moss, M. S., and A. I. Basbaum (1983) The peptidergic organization of the cat periaqueductal gray. II. The distribution of immunoreactive substance $P$ and vasoactive intestinal polypeptide. J. Ncurosci. 7: 1437-1449.

Moss, M. S., E. J. Glazer, and A. I. Basbaum (1983) The peptidergic organization of the cat periaqueductal gray. I. The distribution of immunoreactive enkephalin-containing neurons and terminals. $J$. Neurosci. 3: 603-616.

Nemeroff, C. B., A. J. Oskahn, III, P. J. Manberg, G. N. Erwin, and A. J. Prange, Jr. (1979) Alterations in nociception and body temperature after intracisternally administered neurotensin, $\beta$-endorphin, other endogenous peptides and morphine. Proc. Natl. Acad. Sci. USA 76: $5368-5371$.

Quirion, R., P. Gaudreau, S. St. Pierre, F. Rioux, and C. B. Pert (1982) Autoradiographic distribution of ${ }^{3} \mathrm{H}$ neurotensin receptors in rat brain: Visualization by tritium sensitive film. Peptides 3: 757-763.

Reynolds, D. V. (1969) Surgery in the rat during clectrical analgesia induced by focal brain stimulation. Science 164: 444-445.

Romagnano, M. A., and S. A. Joseph (1983) Immunocytochemical localization of $\mathrm{ACTH}_{1-39}$ in the brainstem of the rat. Brain Res. 276: $1-16$.

Sandkuhler, J., and G. F. Gebhart (1984) Relative contribution of the nucleus raphe magnus and adjacent medullary reticular formation to the stimulation in periaqueductal gray of a spinal nociceptive reflex in the pentobarbital-anesthetized rat. Brain Res. 305: 77-87 (appendix 5).

Steinbusch, H. W. M., and R. Nieuwenhuys (1983) The raphe nuclei of the rat brainstem: A cytoarchitectonic and immunohistochemical study. In Chemical Neuroanatomy, P. C. Emson, ed., pp. 131-207, Raven, New York.

Triepel, J., and C. J. P. Grimmelikhuijzen (1984) A critical examination of the occurrence of FMRFamide immunoreactivity in the brain of guinea pig and rat. Histochemistry 80: 63-71.

Uhl, G. R., and S. H. Snyder (1976) Regional and subcellular distribution of brain neurotensin. Life Sci. 19: 1827-1832.

Uhl, G. R., R. R. Goodman, M. J. Kuhar, S. R. Childers, and S. H. Snyder (1979) Immunohistochemical mapping of enkephalin containing cell bodies, fibers and nerve terminals in the brain stem of the rat. Brain Res. 166: 75-94.

Watanabe, T., Y. Taguchi, S. Shiosaka, J. Tanaka, H. Kubota, Y. Terano, M. Tohyama, and H. Wada (1984) Distribution of the histaminergic neuron system in the central nervous system of rats; a fluorescent immunohistochemical analysis with histidine decarboxylase as a marker. Brain Res. 295: 13-25.

Yaksh, T. L., J. C. Young, and T. H. Rudy (1976) Systematic examination of the rat brain sites sensitive to direct application of morphine: Observation of differential effects with the periaqueductal gray. Brain Res. 114: 83-103.

Young, W. S., III, and M. J. Kuhar (1981) Neurotensin receptor localization by light microscopic autoradiography in rat brain. Brain Res. 206: 273-285. 